\title{
Learning About the Relevance of Education Through an Introduction of the Scientific Method
}

\author{
Juan Pablo Fernández Ramos \\ Basic Research, Ciemat, Madrid, Spain \\ Email address: \\ juanpablo.fernandez@ciemat.es \\ To cite this article: \\ Juan Pablo Fernández Ramos. Learning About the Relevance of Education Through an Introduction of the Scientific Method. Science \\ Journal of Education. Vol. 9, No. 5, 2021, pp. 170-176. doi: 10.11648/j.sjedu.20210905.14
}

Received: August 23, 2021; Accepted: September 17, 2021; Published: October 29, 2021

\begin{abstract}
The education is one of the most valuable, needed and supported aspects in human life. Realizing about the relevance of education can be done in many ways. In this paper, the author reflects one way: through an introduction of the scientific method to students. The scientific method has been introduced to pupils before high school and the qualitative results of it in terms of attention and comprehension, along with the method itself, is treated as well in this paper. In terms of attention from the pupils, a much better connection with them is achieved when the students take part in the class through questions to them or through participation in experiments along with the words and explanations needed. The same education will not be more efficient if it is not more participatory. From the human and social point of view, education is the key to mature as a society. Therefore a very important effort should be taken to not only develop the academical background, but also the human one. Learning moral and self-criticism is more than essential for us to grow as human beings: We are alone; no matter how much we are accompanied, we are the ones who live in this human body and the ones who have to take the ultimate decision whether to do this or not, to be or not to be, to do or not to do, and more than the decision itself what matters is to understand that there is a responsibility along with such a decision.
\end{abstract}

Keywords: Scientific Method, Education, Observation, Hypothesis, Experiment, Quantum Mechanics, Teachers, Participation of Pupils

\section{Introduction}

The education is one of the most valuable, needed and supported aspects in human life. It is clear that the society does not take it for granted and employs lots of human resources to the final purpose of giving each individual the opportunity to have access to the knowledge of different subjects and at levels deep enough for them to understand and grow in all aspects of life but mostly academically $[1,2,3]$. Are we doing enough, is there room for improvement? The scientific method has been carefully treated and presented in an extended way in the literature $[4,5]$. The fundamental connection between the scientific method and the research is applied elsewhere $[6,7]$ and possible roadmaps for evolution are suggested here [8] as well as the interplay between the scientific method and education [9] or the role that the scientific method should play in education [10]. In this paper, the scientific method has been introduced to pupils before high school and the qualitative results of it in terms of attention and comprehension, along with the method itself, are treated. No quantitative results are derived and this might be the purpose of future long term research with more statistics in terms of more schools participating and more input from the students. The purpose of this paper twofold: first to establish a set of optimal conditions that can improve the way can we learn any subject and second to stress the need of more moral input in the education itself, not as a subject, but a transversal and essential content in all the subjects. An example that this is not impossible is given in the study depicted in this paper in which moral and scientific method are connected in many ways, from the same fundamental meaning to the same final goal. After a brief introduction towards understanding the implications of teaching in ??, the motivation and its development are presented in the main body 2: the main idea of the study is shown in 2.1 while how this particular study took place is the argument of sections 2.2,2.3 and 2.4. Unexpected lessons 
learned, final remarks and conclusions are given in 3, 4 and 5 respectively.

Let us think about the quantum effect of the double slits experiment [11]. You all might be aware of it. It consists of a laser that is directed towards and passes through two thin slits (typically $<1 \mathrm{~mm}$ wide) close to each other $(<100 \mu \mathrm{m}$ apart) and a final screen to see the effect. The initial beam of the laser, splits into two when passing through the thin slits (like when in the water the initial wave passes through an object and splits into two). The quantum effect consists of the superposition (the proper word is interference) of the two beams onto the screen showing a clear pattern: regions with no sign of beam (this is the result of the destructive superposition of the two beams) interspersed with regions with some sign of the beam (constructive superposition). Whenever you think of a quantum state or whenever you see the effects in real life of such a quantum effect (in the case of the double slits experiment the effect seen is a pattern of interference between the waves that pass through both slits) you see what is there without you interfering on the quantum system. If you want to get closer to see what happens you start to interfere with the system and the interference pattern disappears and the classic, just one beam on the screen, appears. By interfering, the system is forced to make a choice. Now let/s think about us. We are like (of course this is just metaphoric) wild beams passing through slits in a quantum way. But when a person interacts with us, a choice has to be made. The interaction has changed us and we are not what we were before the interaction. In a positive or negative way education is dramatically essential and important since it might change peoples lives and decisions will be taken by pupils that will not be irrelevant but may be the other way around (it us up to them of course). The teachers, when interacting, we give the pupils the opportunity to make a choice (in real life they have the option of not paying attention and not interacting). The way teachers interact with the pupils is not irrelevant since it might help them to make the proper choice: the most appropriate for them (not for us of course). Three main concerns should be stated clearly to start with:

1. Nothing is fixed: even if not mentioned anywhere else in the text, everything under this article holds a developing idea and the author is trying to find the most appropriate possible way to continue advancing towards its improvement.

2. Besides the fact that nothing is fixed, nothing is demonstrated to work $100 \%$ : circumstances will always make the conclusions derived here different and probably even not applicable there.

3. Nothing is new. The author is sure that this has been studied and tried in different scenarios and using different methodologies. Although everything is invented, not everything is totally and irremediably defined. What is being done here is helping on defining what probably has been already invented or tried elsewhere (this must have been tried already given the many years and experience in the field from many profesionals).
4. This method harks back to Socratic maieutic or to Nicolas de Condorcet: learners are guided, by careful questioning with a view to progressive, logical reflection on the basis questions, reformulations and objections [12].

\section{The Motivation and Its Development}

A study has been carried out with the participation of 13 and 14 years old pupils at school. The study took place within the format of a workshop with the main idea of introducing the scientific method. The study was carried out at two different schools and in a total of 8 courses. Each course received 3 sessions but the number of sessions can be changed according to needs, and circumstances and convenience. The point is that sessions 1 and 3 were done in a certain way and different from 2. Session 2 consisted on the usual way of going through a talk/subject with a speaker/professor taking all the time needed to explain the basics and ideas with almost no stop or any interruption from the pupils. Sessions 1 and 3 were more participatory from the very beginning. In some cases the pupils took the lead in such a way that, even if the purpose of learning holds, the flow changed and focused on other parts of us as scientists (how the author became a scientist). Afterwards, a survey was performed to pupils and teachers and the conclusion reached was as follows. Despite the fact that the novelty and interest of the issues discussed was the same in all the sessions, a much better connection between the professor and the students was achieved in sessions 1 and 3 than the standard session 2. Results: In terms of attention from the pupils (as expressed by them in an afterwards questionnaire).

1. The connection achieved in sessions 1 and 3 (throughout the duration of the whole session) between the professor and the students was $>90 \%$.

2. The connection achieved in session 2 (throughout the duration of the whole session) between the professor and the students was $\approx 50 \%$.

\subsection{Behind the Developing Idea}

The main idea is to explain the three basic pillars of the scientific method: observation, reflection and verification. To help the student to fix the ideas behind each pillar, a set of stories, practice and experiments are carried out. In the way this is done, students not only memorize the basic pillars but also work around them in many different ways so that memory is not needed in fact since it is part of their own experience. They learn through experiencing/practicing/getting involved with the professor through the basic pillars. Aside note: in fact a session for exercising each pillar would be desirable, or even more than one. The more the time given to the pupil to absorb the ideas, the better. Who said that time is limited? For what? For learning time should never be limited. When time intervenes, the curve of learning and the amount of students that follow drops dramatically. Time is always there, but one should interact with the pupils as if there were an unlimited 
amount of time. This is one of the greatest challenge for teachers that are aware of it and have to face it every single day at school. The practice helps to strengthen the pillars. So, once again, it is not the lack of capacity, experience, wisdom of the teachers and it is not the bad behavior or the lack of interest or potential of the students. The key is an important variable in all aspects of life but fundamental in physics: time. The academic program is too long to teach and little time is given to teachers and students to enjoy, share experiences and learn from each other to grow as human beings. Yes, both, the author is also learning a lot in this project. The academic growth of the students is important, but is it really more important than growing as a human being (yes, that is done at home)? But the fact is that days 1 and 3 were different from day 2 (closer to what the author usually does: concept after concept almost without rests) and it was the heaviest for them and the day they learned the least.

\subsection{Session 1}

Two clarifications are always needed from the very beginning:

1. This is outreach, not science. The author uses no formulas, no numbers; just words (initially). Pupils should take all the examples, metaphors and stories mentioned with a grain of salt since they are far from being true, but they are given with the idea to bring the basics closer together and to help the student to understand these basic concepts (like the metaphor of the double slit experiment and professors helping to make the most appropriate choice for them, the pupils).

2. There is always a motivation/attempt to manipulate the students. In this particular case, the author was trying to manipulate them so that those of them with an initial interest, get more interested and, in the end, would decide to come to the research institution and become $\mathrm{Ph} \mathrm{D}$ students in the group.

The first pillar of the scientific method is observation,i.e. to look around and note/write all things that are thought to be relevant in the given/particular/current research/study under consideration. The more annotations the better.

Each concept was explained with two examples: one in a story style and the other by participating in an activity. The fact is that the example/story about the observation was to go back to the XVI century with the heliocentrism and geocentrism.

To explain the relative movement of the sun around the earth there were two main hypothesis: either the sun is in the center or the earth is in the center. The students counted how many objects / observations that each of the groups of scientists used to justify their hypothesis. Geocentrism: sun and moon. Heliocentrism: sun and planets.

1. First moral of the story learned: the more observations the better to strengthen a given hypothesis.

2. Warning that was learned: beware of those special observations that might confuse us, but they are far from being false (i.e.,the special case of the moon as the only object orbiting around the earth). Usually, ahead in time, there is always a new theory that brings all true observations together (gravity: first Newton, then Einstein).

3. Another moral: it is better to face everything as a team, as if the geocentrists were part of our team (because what if, no matter how unimportant it was, the others are right about something or what if we are the ones with a wrong idea?) a team of scientists trying to find out something with two hypotheses but all in the same team/boat.

4. Okam's razor: the geocentrists had to make a movie to explain the observations of the planets moving from the earth backwards and forwards. What explanation would you choose? The simple one or the complicated one?

The second pillar of the scientific method consist of taking all the input gathered, thinking about it and trying to come up with something/a-reason that explains what has been observed: the development of a model, of a hypothesis that might turn into a theory if it is proven to be right. The third pillar is the one that confirms if a hypothesis is right or wrong. In this last step experiments are performed in order to prove or discard a given hypothesis. The moral here is: the more experiments/measurements to prove or discard a given hypothesis, the better. The first session ended with the class performing an experiment to prove / discard the following. The author took a glass with no liquid and asked whether the glass was empty or not. Two groups were formed depending on each pupil/s internal conclusions/thoughts (after discussion between all of us... most of the time the author was alone on one group just for fun... these guys were too clever).

A glass without water and a jar with water were used, and two different experiments took place. The first experiment consisted on just filling the glass with water from the jar in the normal way (with the jar on the top and glass at the bottom). The glass was filled with water. Both hypotheses were still valid. In other to maintain the one of the glass being filled with air, pupils had the problem of an additional explanation given bellow (going against Okam/s razor but anyway... the author was just challenging them). Another experiment was still needed, this time one that would help the class to discard one of the two hypotheses. Again the class proceeded to fill the glass with water but in this new (second) experiment the glass was put upside down and was approached to a jar of water. The ones supporting the hypothesis of the glass being empty, said that what was going to happen is that the glass was going to be filled with water. That did not happen. One by one they did it, and they checked it themselves. The glass, when submerged like this, does not get filled with water. The author explained to them that, although one cannot see it, the glass had air and that is why the water could not enter when the class tried push the air all at once. A pupil said that the first experiment, not trying to fill the glass upside down but fill it up with water, worked still proving the other hypothesis. The author told the pupil that when the water is poured in and at the same time the air is allowed to go out (which is what normally happens, like in the first experiment) then the glass fills up. In the last experiment, the water tried to get in the glass 
suddenly but not leaving space for the air to go away.With the first experiment both options were still valid; with the second, one of them was discarded. Without air, the glass should have been filled in any scenario/experiment performed and that did not happen. Again, the more experiments the better. A bottle filled in half by liquid is a half-full or half-empty bottle? The moral here is that there is not a unique answer. May be the answer is both options (always filled with the two things in the proportion given by the circumstances) or even another one that is not told us. Matter is hollow (almost a desert) as Rutherford demonstrated [13].

\subsection{Session 2}

Next day, as mentioned, the session was more formal, but the author also tried to do it as enjoyable as possible. The class went through our constituents from the biggest in size to the smallest (organs, tissues, cells, molecules, atoms, and the subatomic world). Some attention was paid to some aspects of this subatomic world. One of them for example was the meaning of elementary particle. To a scientist, rather than unbreakable or the smallest particle, it means a fundamental particle, a particle the explains how other more complex ones are built or constructed from first principles (the first principles behind the theory that gives rise to the fundamental particles). A quark is elementary not for being a point-like particle but for being one of the fundamental particles of the so called Standard Model of particle physics $[14,15,16,17]$ Scientists do not usually say that a quark is the smallest particle. Scientists prefer more often to say that within the ranges of energy in which that particle has been proved, there is no evidence of internal structure. The second does not necessarily mean that it does not have internal structure and therefore is point-like, but that, within the range of energy tested so far, no evidence of structure is seen. That is all. Elementary as fundamental is what matters more than the size of the particle (point-like or not). As another example, a proton (with its internal structure of quarks and gluons) seems like a fundamental particle to an electron that sees the same but opposite electric charge so that it is fine for both to accept the contract written in an electromagnetic language or using the electromagnetic fundamental force, one of the four major forces as mentioned bellow. It is the interaction of the particle with the world (or with the experiment) what allows us to recognize, measure and categorize it. The class went through the main four types of interactions: gravitational, weak, electromagnetic and strong force. Certain peculiarities of each force in terms of intensity, the way that particles communicate in each case/scenario/force-beingused to communicate, etc., were given, including trying to understand one of the paradoxes of this universe:

1. Forces are different in intensity and ordered in this way (from strongest to weakest): strong, electromagnetic, weak, gravitational.

2. There is something intrinsic in each particle that allows that particle to talk, interact in such a language (like the electromagnetic charge allowing electrons and proton to make an electromagnetic contract and form the atom of hydrogen). These are generically called intrinsic charges being the electromagnetic charge one example. The color is the equivalent intrinsic charge associated to the strong force.

3. Particles can have several intrinsic charges (like the identity card reflecting several intrinsic aspects or the DNA) and therefore ways to to interact/talk.

4. The fact that at different scales (the author is referring to the spatial scale now) different forces hold regardless of their intensity does not necessarily mean that there is no explanation for it: at different scales, despite their intensity, different forces take the lead (e.g. the strong force only plays a role at very short scales of the size of the proton, $f m$ or $\left.<10^{-15} \mathrm{~m}\right)$. At bigger scales like the size of the atom ( angstrom, or < $10^{-10} \mathrm{~m}$ ) the electromagnetic force takes the lead. Then, at much bigger scales, like the scale of the planets since there is roughly the same amount of positive and negative electromagnetic charges, the planets are typically (except for some special cases) neutral so gravity wins (the author is omitting here the reason why the strong force has so small range of reach and not mentioning the weak force just for simplicity though it is essential to understand how the sun works from the point of view of the sun as a source of energy but not from the point of view of the sun binding all planets together around it).

Therefore we live in a universe in which the weakest (in terms of intensity) of the forces is the one that drives, sculpts it at large (cosmological) scales. It can be seen that different forces dominate at different scales. Besides, the peculiarities / laws of physics do not necessarily have to be the same at different scales either. The only example given in this session was to use a coin in order to understand just one of the special features of the quantum world in which those elementary particles live. Particles have states, i.e., they have different ways to manifest themselves to the world (like one can manifest to the world with a happy or sad face or with anger or not-anger: good/evil human state). The funny thing of this quantum world is that the particles are combination of those states and it is only when one asks (interacts with the particle) when the particle shows itself as this or that state. The example of the coin was quite illustrative since when you flip the coin it is in both states like the elementary particle being a combination of the two faces of the coin at the same time. It is just when the coin stops (falls in the hand or into the ground) when the coin shows itself as head or tail. A rock is a rock and no matter if look at the rock now or later, the same (except if we wait ages) structure/answer will be observed. Human beings are something special in the sense that humans might resemble those particles living in the quamtum world. Sometimes humans act as angels sometimes as demons or beasts (metaphor again and too simplistic but the main concept is there). The fact that humans show theirselves in one way or the other (with one state or the other) depends on their own internal characteristics (sleep, hunger, etc) and also how they 
couple-with/adapt-to the given circumstances that surrounds us (this is called boundary conditions in the quantum world). There were other peculiarities of the quantum world being introduced but with little / no participation of the pupils. The final part of the session was mentioning a story about a tiger that grew up with lambs. Then another tiger showed up and demonstrated that it was not only a fact that the first tiger was similar in appearance and also could do things similar that never tried like roaring, but it was also a fact that the two tigers were the same type of animal and different from the lamb. Then the story ends encouraging you to forget about the lamb and roar like the tiger you really are. This is the moment that the author liked the most. When going through sessions 1 and 2, the class saw that there is not only a unique answer or the type of answer with the two options they want to sell us. We can be both and in fact the author is more tempted to say that both states are present and humans manifest as one or the other depending on of internal and external conditions and how both (humans and boundary conditions) couple/combine. Sometimes stories might be tricky, and the class has to be aware of the people telling that story and their intentions. The first thing the author did in session 1 was telling them my intention, the purpose, the reason why the author wanted to manipulate them. Despite the roaring of my tiger or beast this session was not as accepted/followed as 1 and 3 .

\subsection{Session 3}

It was time to practice. In particular, regarding the part of the practical exercise related to the observation, it was time to close the eyes. Different sounds were made and students had to identify which object it corresponded to or the type of material and the amount of objects $(<10,10-20$ or $>20)$. The author used a violin without the students knowing what it was. First the author played it with my fingers (not with the bow) plucking several strings at the same time. It reminded them of a guitar more than a violin and the author did it on purpose. They talked among themselves and said that it could not be a guitar (all this with closed eyes) because it could not fit in the box the author brought... It's over now but the idea was (another session) in which (the author would tell them) they realized that they had fallen into the geocentric trap. The observation of the size of the box was real but it was misleading, as well as the sound (which reminded them of something from the past experience) and you can not imagine the amount of ukuleles that the author saw that they wrote (blindly too). Why in another session? Because that is when it would be necessary to think on the relationship between observation and interpretation and the danger of misinterpretation. It is easily forgotten that these interpretations are not reality and it is essential to make sure in every possible way that the conclusion taken, the interpretation is not biased by the way the observation is being made or by previous prejudices (like the size of the box... or that the instrument sounded more like a guitar with which they discarded any other option and therefore prevented them from using other observations to make the final conclusion). The more observations the better... but two are not always enough... The author could give more details of what was done then and also of the surprises the author got from the students... participation, involvement, trust, joy and amazing faith. For example the author did not use audiovisual media at all and it worked fine: the author was going from one experiment to the other... from one exercise to another exercise... from moral to moral... and in constant interaction with them, so that sometimes the class got to change all the script.

\section{Beyond the Scientific Method: Unexpected Sessions}

\subsection{Sacrifice vs Fun}

We need hobbies. They are an essential leg for us. Again, although everything is invented, not everything is totally and irremediably defined. What should a teenager of that age do? have fun or sacrifice in favor of his/her future (because he will have time for the last thing once his academic training is advanced)? And what if the problem is to see it as different things when maybe both can be given at the same time? There are disciplines (art, music, sport) that teach you just that: with effort and sacrifice you can see, little by little, the result and enjoy what has been achieved by getting better and better at playing an instrument, running longer and longer, drawing better and better. The educational system is a key in the sense of helping the students to realize that both things can be the same. Do not just run, run and realize, after a few weeks, what you have achieved (same for playing instruments, drawing or other arts and crafts). Understand that there are ways to find both things together, sacrifice and fun. And something even more important. Those choices, becoming a runner or a musician or any other artist, will become an essential leg in one/s life that will complement the professional/academic perspective/leg. The more legs one has to lean on, the better to avoid falling down when one temporarily breaks. From the academic point of view, doing sports, playing instruments is not something irrelevant at all, but the other way around. Once you take it as a habit / hobby you will see that, after running a bit, the body is more prepared to absorb, learn and experience in the other world (the academic one). Both worlds complement each other.

\subsection{The Good and the Evil}

At a certain point in a course, we came to wonder if there was good and evil. The answer of the pupils left the author puzzled to see these young boys with that critical and reflecting (meditating) spirit so early and so successfully. The author fully agree with them. There is neither good nor evil. It is not written in the genetic code, but we better agree and define it to be able to organize and advance / evolves as that entity called society. It is the organizational and associative character that makes us progress as everything, individual and species. All the teachers, the scientists and all the students, all of us 
are an example of what those pupils (Ismael and Samuel) said. Samuel was rather on the side of the protection of the tribe without care about the other tribes and Ismael on the side that if we did not agree on what is right and wrong this would be chaos. And the author was on everyone's side because to evolve as a society we not only have to organize and take care of the tribe but go one step further and take care of that whole system. And the author? How is the author going to take care of that whole as an individual? It is impossible ! The author can not do it alone, but the answer is in all the teachers and then in each and every one of those formidable students. It is not impossible to reach this goal, through our association, the mutual interest in training / education in values and the passion for science, the arts (that music that calls me from time to time), knowledge in general, sports, etc, and above all for our selfless devotion and dedication.

\section{Remarks}

We are that weird. The greatest wonders of the world are in front of us (i.e. they are part of us already). The greatest miracles in the world are made by us (i.e., by our body) and we are like celestial beings that do not realize it. We live in this heaven called Earth, and we pass by as if it were completely normal to live like this. The very fact of reorienting the air molecules in such a way that all of them go in rhythm and arrive at our hearing at certain frequencies (what we normally call speaking) seems to us the most normal thing in the world when, to a caveman, it would seem that we are possessed and that we are Gods. I don't know how I did it, or how I got/learnt it, but the fact is that when I was little, by imitation above all, I saw others doing it, I discovered that I also had a speech apparatus (vocal cords) with which to produce similar sounds and begun to play and practice. And here I am, typing but I can say this myself using my voice. Me and many like me. ${ }^{1}$ On the other hand, other phenomena that our body can produce but does not since we have not had the opportunity to learn them by imitation, and that in some people their body produces, seem most extraordinary to us. Writing music is an example. I write music but I do not feel special, it is just that I learnt by imitation as everybody would do if they had the chance (as I said, you need the special apparatus and ears as essential). Our neurons can dance to the same tone (same frequency), they can enter in resonance like the air molecules do and such an ensemble is being produced by something similar to our vocal cords, there, in some part of that wonderful and complex organ called the brain. The fact that you think you do nothing does not mean that you are not really doing it because doing nothing can precisely mean allowing that the brain sends our neurons to dance in a certain way. We know we can talk to anybody and we use to talk to us in the form of thoughts that help us to take a more optimate decision. Imagine learning and allowing us to think or express ourselves in many other ways... The explanation is the least, the wrapper, the words used to explain it, are the least, but the phenomena that we observe have an explanation, regardless of whether we have seen them today for the first time in our lives, or we have been using it (like our voice) since 9 months old (or some even before). It is our ability to wonder that self-suggests and opens our minds and prepares us for experiencing what seems impossible to us because we have never experienced it before... almost like talking... Someday I will stop moving my toes and it will be when I realize that what I was doing was unbelievable. Human beings are so incredibly awesome that they even forget about it. This is not narcissistic in my humble opinion, it is to give each thing its proper relative weight, both to the known and to the unknown (the uncommon or unknown does not necessarily have a higher weight than the everyday things that happen commonly in our lives). The fact that things will always be in a certain way is taken for granted and that is a quite commonly extended mistake. At a certain temperature (called critical temperature) a ferromagnetic material stops behaving as such and certain metal ceases to offer resistance to the passage of electrons (superconducting material is normal material at room temperature). Not everything is how we think it is forever, not even the time and space that we share right now. That does not mean that tomorrow everything can be anything completely different. No. It may be different but not completely different since there are some laws of physics that are absolute in the sense that they are the same regardless of the conditions (for example, the speed of light in a vacuum is always the same regardless of the reference system in which you measure it, i.e. regardless of their motion relative to the source of that light). And knowing that what is known is as surprising as what is to be known, can help us to best interpret what we observe. Never stop observing what surrounds us. Interpreting is inevitable due to issues that we already know about survival (if I do not change direction when I see a column I will crash into it) but let's try to use other light in addition to the one we always use when we observe what we observe, just in case that allows other interpretations to be found. Moreover, the major mistake is to consider what is observed by us as the reality instead of an interpretation of reality for us to survive. For example, there are other frequencies in the electromagnetic spectra covered by the light of the sun, that our eyes do not see, but they are there. If another external device catches/observes them (e.g. a camera sensitive to the infrared light), our imagination goes to the roof on making interpretations that are not necessarily the correct ones. The correct one being that it is still nature, it is still light, the nature (light) we notice neither with our eyes nor with our common sense. And speaking of light, the light we have at home, what is it? That light that makes the television work, the refrigerator? is it pure science or is it a miracle? Both, a miracle of science.

\section{Conclusions}

Education is the key to mature as a society. There are several main aspects that are worthwhile stressing here about this.

1. The same education will not be more efficient if it is not

\footnotetext{
${ }^{1}$ Apart from the vocal cords we need our ears to verify what we are saying and not move blindly.
} 
more participative.

2. Given the current academic program, the teachers do their best. Why can we not let them decide which is the best way to educate the pupils?

3. A law of education without the full participation (i.e. made by them) of the teachers is suboptimal.

There is no unique way to teach, nor to learn. Each one should find his own way and, once each individual has reached this goal, the society will be plural and rich.

\section{Acknowledgements}

Thank you very much to teachers in general and those of the school Las Rosas in particular (Madrid, Spain). Their role in this society is essential as already mentioned: $O n$ many occasions I (the current writer/speaker) already knew this because many told me, only that I forgot, and it turned out like that and the teachers realized it and also made me notice it. Sometimes I do learn and unlearn but the best thing is that you are there to (as I always say) get the best version of me. Special thanks to Mercedes Alós Cortés who gave me the opportunity to start this magnificent project and to my colleagues at CIEMAT institute, ZEUS, CDF and CMS collaborations that shared with me all their knowledge and what goes beyond the knowledge.

\section{References}

[1] C. Fu and X. Feng, Workshop Physics: An ActivityBased Curriculum Enhanced by Computer Tools, Science Journal of Education 9 (3), 115-123 (2021).

[2] C. Zhang and L. Zhao, Education and Cultivation

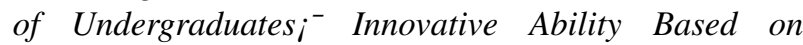
Philosophical Thinking, Science Journal of Education 9 (1), 1-5 (2021).

[3] Y. Li and X. Wang, Online Teaching of College Physics with Multi Teaching Methods and Temperature, Science Journal of Education 9 (2), 27-31 (2021).

[4] D. S. Fudge Teaching the Scientific Method Using Current News Articles, The Journal of Experimental Biology 217, 1202-1204 (2014).

[5] E. O. Voit, Perspective: Dimensions of the scientific method, PLoS Comput Biol 15 (9), e1007279 (2019).
[6] M. Castillo, The Scientific Method: A Need for Something Better? American Journal of Neuroradiology 34 (9), 1669-1671 (2013).

[7] L. K. Palmer and C. G. Mahan, Teaching the Scientific Method Using Current News Articles, The American Biology Teacher 75 (5), 355-356 (2013).

[8] S. Carrol, Defining the scientific method, Nature Methods 6, 237 (2009).

[9] J. Sjöström and I. Eilks, The Bildung theory; ${ }^{a}$ From von Humboldt to Klafki and beyond, In Akpan \& Acuna (Eds.), Science Education in Theory and Practice (pp. 55“C67). Springer International Publishing (2020).

[10] M. Emden, Reintroducing the Scientific Method to Introduce Scientific Inquiry in Schools?, Sci \& Educ 30, $1037{ }^{\circ} \mathrm{C} 1073$ (2021).

[11] T. Young, The bakerian lecture: experiments and calculation relative to physical optics, https://royalsocietypublishing.org/doi/pdf/10.1098/rstl. 1804.00011803.

[12] Social and H. S. S. (UNESCO), Teaching philosophy in latin america and the caribbean, https://unesdoc.unesco.org/ark:/48223/pf0000185119 2009.

[13] E. Rutherford, The scattering of alpha and beta particles by matter and the structure of the atom, Philosophical Magazine 21, 669-688 (1911).

[14] W. N. Cottingham and D. A. Greenwood, An Introduction to the Standard Model of Particle Physics, Cambridge University Press, 2007.

[15] D. Coleman, D. Kaiser, A. S. Wright, Theoretical Physics in Your Face Selected Correspondence of Sidney Coleman, World Scientific Publishing Co Pte Ltd (2022).

[16] H. Schopper, Particle Physics Reference Library, Springer (2020).

[17] D. Griffiths, Introduction to Elementary Particles, WileyVch (2008). 